\title{
Prefrontal cortex miR-29b-3p plays a key role in the antidepressant-like effect of ketamine in rats
}

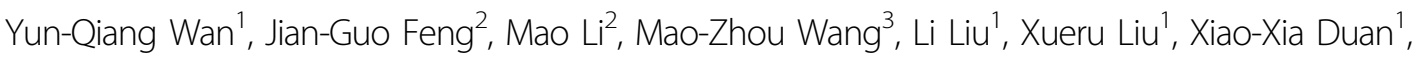 \\ Chun-Xiang Zhang ${ }^{4}$ and Xiao-Bin Wang ${ }^{1}$
}

\begin{abstract}
Ketamine has a rapid, obvious, and persistent antidepressant effect, but its underlying molecular mechanisms remain unknown. Recently, microRNAs (miRNAs) have emerged as important modulators of ketamine's antidepressant effect. We investigated the alteration in miR-29b-3p in the brain of rats subjected to ketamine administration and chronic unpredictable mild stress (CUMS), and a sucrose preference test and forced swimming test were used to evaluate the rats' depressive-like state. We used recombination adeno-associated virus (rAAV) or lentivirus-expressing miR-29b-3p to observe the change in metabotropic glutamate receptor 4 (GRM4). Cell culture and electrophysiological recordings were used to evaluate the function of miR-29b-3p. Ketamine dramatically increased miR-29b-3p expression in the prefrontal cortex of the normal rats. The dual luciferase reporter test confirmed that GRM4 was the target of miR-29b$3 p$. The miR-29b-3p levels were downregulated, while the GRM4 levels were upregulated in the prefrontal cortex of the depressive-like rats. The ketamine treatment increased miR-29b-3p expression and decreased GRM4 expression in the prefrontal cortex of the depressive-like rats and primary neurons. By overexpressing and silencing miR-29b-3p, we further validated that miR-29b-3p could negatively regulate GRM4. The silencing of miR-29b-3p suppressed the $\mathrm{Ca}^{2+}$ influx in the prefrontal cortex neurons. The miR-29b-3p overexpression contributed to cell survival, cytodendrite growth, increases in extracellular glutamate concentration, and cell apoptosis inhibition. The overexpression of miR29b-3p by rAAV resulted in a noticeable relief of the depressive behaviors of the CUMS rats and a lower expression of GRM4. The miR-29b-3p/GRM4 pathway acts as a critical mediator of ketamine's antidepressant effect in depressive-like rats and could be considered a potential therapeutic target for treating major depression disorder.
\end{abstract}

\section{Introduction}

Major depressive disorder (MDD) is a common chronic mood disorder that results in heavy social and economic burdens ${ }^{1}$. By 2020, MDD is expected to be the second leading cause of disability worldwide ${ }^{2}$. However, to date, the prospect of antidepressant treatment is poor. In addition, traditional antidepressant drugs require a few

\footnotetext{
Correspondence: Xiao-Bin Wang (wangxiaobin67@163.com)

${ }^{1}$ Department of Anesthesiology, The Affiliated Hospital of Southwest Medical University, Luzhou, Sichuan Province, People's Republic of China

${ }^{2}$ Laboratory of Anesthesiology, The Affiliated Hospital of Southwest Medical

University, Luzhou, Sichuan Province, People's Republic of China

Full list of author information is available at the end of the article.

These authors contributed equally: Yun-Qiang Wan, Jian-Guo Feng, Mao Li.
}

days or weeks to achieve their antidepressant effect. The main reason for these inadequacies in the treatment of depression is that a comprehensive understanding of the pathogenesis of depression is lacking. Ketamine is a $N$ methyl-D-aspartate receptor antagonist. Accumulating evidence from clinical and basic research shows that ketamine exerts a quick and persistent antidepressant-like effect $^{3-7}$. Compared with traditional antidepressant drugs, the antidepressant effect of ketamine is obvious and quick. Due to its clear and rapid antidepressant effect, ketamine has become a topic of interest in the study of depression. However, the functional mechanism of ketamine still remains unclear.

\section{(c) The Author(s) 2018, corrected publication 2022}

(c) (i) Open Access This article is licensed under a Creative Commons Attribution 4.0 International License, which permits use, sharing, adaptation, distribution and reproduction cc) in any medium or format, as long as you give appropriate credit to the original author(s) and the source, provide a link to the Creative Commons license, and indicate if changes were made. The images or other third party material in this article are included in the article's Creative Commons license, unless indicated otherwise in a credit line to the material. If material is not included in the article's Creative Commons license and your intended use is not permitted by statutory regulation or exceeds the permitted use, you will need to obtain permission directly from the copyright holder. To view a copy of this license, visit http://creativecommons.org/licenses/by/4.0/. 
miRNAs constitute a class of small non-coding RNA molecules whose function is to regulate the transcription and translation of downstream-related genes. Furthermore, recent studies suggest that miRNAs are associated with the pathophysiology of depression and could be potential targets for novel antidepressant treatment ${ }^{8-11}$. The miR-29 family members have been shown to mediate neuropathological processes in response to environmental stress factors ${ }^{12}$ and have been suggested as potential biomarkers for the diagnosis and prognosis of neuropsychiatric disorders ${ }^{13}$ and relevant neurodegenerative diseases ${ }^{14-17}$. In our previous report, the acute administration of ketamine resulted in the abnormal expression of various miRNAs (including those belonging to the miR-29 family), which was involved in the antidepressant effect of ketamine $^{18}$. miR-29b expression is elevated in the hippocampus of early-stress rats after acute ketamine administration $^{11}$. Metabotropic glutamate receptor 4 (GRM4) has been predicted to be the target gene of miR29 in bioinformatic analyses. GRM4, which belongs to metabotropic glutamate receptor group III, is mainly expressed in presynaptic membranes of brain neurons. The main function of GRM4 is regulating dopamine, glutamate, tryptophan, and neurotransmitter metabolism, thus affecting neurotransmitter release-mediated neural signaling pathways ${ }^{19}$. In addition, relevant studies have shown that the increased expression of GRM4 is closely related to the occurrence and development of depression; hence, GRM4 may be involved in the pathogenesis of depression ${ }^{20}$.

In this study, we investigated the effect of ketamine on $\mathrm{miR}-29 \mathrm{a} / \mathrm{b} / \mathrm{c}-3 \mathrm{p}$ expression in the hypothalamus, hippocampus, and prefrontal cortex (PFC) of depressed rats and the subsequent regulation of GRM4 expression. Our findings may provide potential therapeutic target genes and open up novel avenues for drug design for MDD treatment.

\section{Materials and methods \\ Ethics statement}

All experimental procedures involving animals were performed in accordance with the NIH Guide for the Care and Use of Laboratory Animals and the Chinese Society for Neuroscience and Behavior Recommendations for Animal Care. The experimental surgeries and treatments were approved by the Ethics Committee of the Affiliate Hospital of Southwest Medical University (approval number Ky2012044).

\section{Subjects and drug treatment}

Adult male Sprague-Dawley rats (150-200 g) were purchased from Chengdu Dashuo Animal Center. The rats were acclimated to the normal experimental environment $\left(12 \mathrm{~h} \mathrm{light/dark}\right.$ cycle, $22 \pm 2{ }^{\circ} \mathrm{C}$ room temperature) for 1 week with food and water available ad libitum. The rats received intraperitoneal injections of 10 $\mathrm{mg} / \mathrm{kg}$ ketamine, which is the dose found by previous studies to be appropriate for producing an antidepressive effect in rats ${ }^{21,22}$. The control rats received intraperitoneal administrations of the same volume of saline. After $24 \mathrm{~h}$, the rats were sacrificed for the miRNA and protein analyses.

\section{Chronic unpredictable mild stress}

The rats were subjected to chronic unpredictable mild stress (CUMS) as previously described ${ }^{23}$. Briefly, the CUMS procedure used in this study involved the following 14 mild stressors: continuous overnight lighting, tilted cage, water deprivation, food deprivation, noise, wet padding, strange objects, no padding, suspension $(6 \mathrm{~h})$, tail pinch, combined cages, shaker stress, exchanging rat cages, and changing the feeding environment. The stressors were randomly performed every day for 35 days, but the same stressor was never applied on two consecutive days. The rats in the control group were housed separately in another room. Depression-related behavioral tests, including the sucrose preference test and forced swimming test (FST), were performed on days 28 and 35 after CUMS. The weight and fur scores were also evaluated as described by Ducottet et $\mathrm{al}^{24}$.

\section{Sucrose preference test}

The sucrose preference test was performed using the method described by Sergio et $\mathrm{al}^{25}$. The data were collected on days 0,28 , and 35 after CUMS. Briefly, the rats were habituated to drink $1 \%$ sucrose solution from two bottles for $24 \mathrm{~h}$. After fasting and water deprivation for the following $24 \mathrm{~h}$, the rats were allowed to drink one bottle of $1 \%$ sucrose solution and another bottle of water freely for $3 \mathrm{~h}$. The water and sucrose consumption were measured, and the preference for sucrose over water (sucrose/ [sucrose + water] $\times 100 \%$ ) was used as a measure of the reduced ability to experience pleasure.

\section{Forced swimming test}

The FST was conducted in a cylinder that was $65 \mathrm{~cm}$ tall and $30 \mathrm{~cm}$ in diameter that was filled $40 \mathrm{~cm}$ high with water $\left(22-23^{\circ}\right)$. The FST was performed for $6 \mathrm{~min}$, and the immobility time (in s) was recorded during the final $5 \mathrm{~min}$. The immobility time was defined as the time during which the rat stood still without struggling, used only essential movements to keep its head above water, or contacted the bottom for more than $1 \mathrm{~s}$.

\section{Cell culture and drug treatment}

Primary PFC neuronal cultures were prepared as previously described ${ }^{26}$. Briefly, the PFC was dissected from an embryonic day 16.5 brain, and the neuronal cells were 
dissociated by trypsin digestion. The cells were seeded in $35 \mathrm{~mm}$ poly-lysine-coated plates at a final density of $1 \times 10^{6}$ cells $/ \mathrm{ml}$ in Neurobasal medium (Gibco) supplemented with B27 and $2 \mathrm{mM}$ GlutaMAX. The cells were maintained in a humidified atmosphere of $5 \% \mathrm{CO}_{2}$ and $95 \%$ air at $37^{\circ} \mathrm{C}$. After $2 \mathrm{~h}$, the Neurobasal medium supplemented with B27 and $2 \mathrm{mM}$ GlutaMAX in the plates was changed to Neurobasal medium supplemented only with B27. The primary cells were allowed to grow for 7 days before treatment and then treated with ketamine at a final concentration of $50 \mu \mathrm{M}$ for $0,1,3,6$, or $12 \mathrm{~h}$. The cells were harvested for the miRNA-29b-3p and GRM4 expression analyses. The rat brain glioma C6 cells and human embryonic kidney 293T (HEK293T) cells were kindly provided by the Stem Cell Bank of the Chinese Academy of Sciences. The C6 cells were maintained in DMEM/F12 medium, and the HEK293T cells were cultured in RPMI-1640 medium. The cells were maintained at $37^{\circ} \mathrm{C}$ in a humidified $\mathrm{CO}_{2}$ incubator in a medium containing $10 \%$ heat-inactivated fetal bovine serum (Biological Industries, CT, USA), $100 \mathrm{U} / \mathrm{ml}$ penicillin, and 0.1 $\mathrm{mg} / \mathrm{ml}$ streptomycin.

\section{siRNA and miRNA mimic transfection}

The small interfering RNA (siRNA)-targeting GRM4 (GRM4-siRNA) and negative control siRNA (NC) were designed and synthesized by GenePharma (China). The rat miR-29b-3p mimic (UUCCCUUUGUCAUCCUAU GCCU) and negative control (UUUGUACUACACAAAA GUACUG) were purchased from RiboBio Co. (China). The cells were transiently transfected with siRNAs or mimics using RNAiMAX Transfection Reagent (Invitrogen, USA) according to the manufacturer's instructions. The cells were subsequently examined $24 \mathrm{~h}$ after transfection.

\section{miRNA target prediction and luciferase assays}

The candidate genes targeted by miR-29b-3p were predicted using the following four miRNA target prediction databases: MicroCosm (http://www.ebi.ac.uk/ enright-srv/microcosm/htdocs/targets/v5/), miRanda (http://www.microrna.org/microrna/home.do), TargetScan (http://www.targetscan.org/), and miRDB (http:// mirdb.org/miRDB/). The genes that were predicted by all four databases and implicated in the pathophysiology of MDD were selected for a luciferase reporter assay.

A dual luciferase reporter assay system (Promega, USA) was used to determine the direct interaction between miR-29b-3p and GRM4. The primers used to generate the GRM4 wild-type 3'-untranslated region (3'-UTR) containing miR-29b-3p-binding site reporter constructs based on pmirGLO (Promega, USA) were as follows: wtGRM4-3'-UTR, forward 5'-AAACTAGCGGCCGCTAGTGTGGCTTGGTGCTGAGGATT-3' and reverse
5'-CTAGAATCCTCAGCACCAAGCCACACTAGCGG CCGCTAGTTT-3'. The primers used for the mutant GRM4 3'-UTR reporter constructs were as follows: mutGRM4-3'UTR, forward 5'-AAACTAGCGGCCGCT AGTGTGGCTCATGTACGAGGATT-3' and reverse 5' CTAGAATCCTCGTACATGAGCCACACTAGCGGCC GCTAGTTT-3'. For the luciferase assays, C6 cells were transiently co-transfected with $50 \mathrm{nM}$ rno-miR-29b-3p mimics or control mimics and $0.5 \mu \mathrm{g}$ pmirGLO containing the wild-type or mutated GRM4 3'-UTR. The luciferase activity was analyzed $48 \mathrm{~h}$ after transfection as indicated by the manufacturer's instructions.

\section{qRT-PCR analysis}

The miRNA-29a/b/c-3p and GRM4 mRNA expression levels were determined using quantitative reverse transcriptase polymerase chain reaction (qRT-PCR) using the mirVana qRT-PCR miRNA Kit (Ambion Life Technologies, USA), according to the manufacturer's instructions. A complementary DNA template generated from $1 \mu \mathrm{g}$ total RNA was used for the qRT-PCR analysis. The miRNA-29a/b/c-3p and GRM4 mRNA amplification and detection were performed using an ABI 9700 Fluorescence PCR System (Applied Biosystems, USA). The primers used for miRNA-29a/b/c-3p, U6, GRM4, and $\beta$ actin in the qRT-PCR analysis are shown in Table 1 . The fluorescence signal was normalized to unify the internal reference level, and the threshold cycle $\left(C_{\mathrm{T}}\right)$ was set in the exponential amplification phase of the PCR. The relative gene expression of miRNA-29a/b/c-3p and GRM4 mRNA was calculated using the $2^{-\Delta \Delta C T}$ method.

Table 1 Sequences of primers used for the real-time quantitative PCR analysis

\begin{tabular}{|c|c|c|c|}
\hline Gene & Direction & Primer sequence & Length \\
\hline \multirow[t]{2}{*}{ Rno-U6 } & $\mathrm{F}$ & TGCGGGTGCTCGCTTCGGCAGC & $22 \mathrm{bp}$ \\
\hline & $\mathrm{R}$ & CCAGTGCAGGGTCCGAGGT & $19 \mathrm{bp}$ \\
\hline \multirow[t]{2}{*}{ Rno-miR-29a-3p } & $\mathrm{F}$ & TGCGGTAGCACCATCTGAAA & $20 \mathrm{bp}$ \\
\hline & $\mathrm{R}$ & CCAGTGCAGGGTCCGAGGT & $19 \mathrm{bp}$ \\
\hline \multirow[t]{2}{*}{ Rno-miR-29b-3p } & $\mathrm{F}$ & TGCGG TAGCACCATTTGAAAT & $20 \mathrm{bp}$ \\
\hline & $\mathrm{R}$ & CCAGTGCAGGGTCCGAGGT & $19 \mathrm{bp}$ \\
\hline \multirow[t]{2}{*}{ Rno-miR-29c-3p } & $\mathrm{F}$ & TGCGG TAGCACCATTTGAAA & $20 \mathrm{bp}$ \\
\hline & $\mathrm{R}$ & CCAGTGCAGGGTCCGAGGT & $19 \mathrm{bp}$ \\
\hline \multirow[t]{2}{*}{ Rno- $\beta$-actin } & $\mathrm{F}$ & AAGTCCCTCACCCTCCCAAAAG & $22 \mathrm{bp}$ \\
\hline & $\mathrm{R}$ & AAGCAATGCTGTCACCTTCCC & $21 \mathrm{bp}$ \\
\hline \multirow[t]{2}{*}{ Rno-GRM4 } & $\mathrm{F}$ & AGTGACAACAGCCGCTATGAC & $21 \mathrm{bp}$ \\
\hline & $\mathrm{R}$ & CACACACCTCCGTTCTCTCG & $20 \mathrm{bp}$ \\
\hline
\end{tabular}




\section{Western blot analysis}

The total protein extracted from the rat cerebral tissue or cultured neurons was used to analyze the GRM4 protein levels via a Western blot analysis. The cerebral tissue and primary neuron cells were lysed using RIPA lysis buffer with phenylmethylsulfonyl fluoride (Beyotime, China). The protein concentration was quantified by a BCA assay (Beyotime, China). Thirty micrograms of total protein from each sample were subjected to $12 \%$ sodium dodecyl sulfate-polyacrylamide gel electrophoresis and then transferred to polyvinylidene difluoride (PVDF) membranes (Millipore, USA). The membranes were blocked in TBST (Tris-buffered saline, 0.1\% Tween-20) with $5 \%$ skim milk at room temperature for $1 \mathrm{~h}$ and then incubated with a primary GRM4 antibody (Abcam, USA) at $4{ }^{\circ} \mathrm{C}$ overnight. After three washes with TBST buffer, the PVDF membranes were further incubated with a secondary horse radish peroxidase-conjugated antibody. The immunoblotting bands were detected via Western Lightning Chemiluminescence Reagent (Perkin Elmer, USA). An AlphaImager analyzer (Alpha Innotech Corporation, CA, USA) was used to measure and quantify the optical density values of the protein bands.

\section{Apoptosis assay}

Apoptosis in the cultured neurons was measured by terminal deoxynucleotidyl transferase dUTP nick-end labeling (TUNEL) staining as previously described ${ }^{27}$. Briefly, hippocampal neurons cultured on coverslips in $24-$ well plates were fixed in $4 \%$ paraformaldehyde. The TUNEL staining was conducted using an In Situ Cell Death Detection Kit (Roche, USA) according to the manufacturer's protocol. The number of TUNEL-positive cells was counted under a fluorescence microscope.

\section{Generation of miR-29b-3p-overexpressing and miR-29b- 3p-silencing lentiviruses and cell infection}

The miR-29b-3p-overexpressing and miR-29b-3psilencing lentiviral particles (lent-over/miR-29b-3p and lent-inhib/miR-29b-3p) were purchased from Hanbio Biotechnology Co., Ltd. (China). Lenti-NC served as a negative control. Briefly, pre-miR-29b-3p was amplified from the genome of a $\mathrm{C} 6$ cell and cloned into the lentivirus expression vector pHBLV-U6-ZsGreen-Puro (Hanbio Biotechnology Co., Ltd., China) with BamHI and EcoRI sites to overexpress miR-29b-3p. The details of premiR-29b-3p are shown in Supplemental figure. The antagonist short hairpin RNA (AACACTGATTTCAAATGGTGCTA) was inserted into the lentivirus vector pHBLV-U6-Scramble-ZsGreen-Puro (Hanbio Biotechnology Co., Ltd., China) to silence miR-29b-3p. Twenty multiplicity of infection lentivirus particles were added to the medium of rat PFC primary neuron cells. The infection effects were detected by immunofluorescence microscopy. The cells were analyzed by qRT-PCR and Western blotting $72 \mathrm{~h}$ after the lentivirus infection.

\section{Generation of miR-29b-3p-overexpressing AAV and stereotaxic surgery}

The miR-29b-3p-overexpressing adeno-associated virus (AAV) (AAV-miR-29b-3p) was purchased from Hanbio Biotechnology Co., Ltd. (China), and AAV-GFP served as a negative control. Briefly, pre-miR-2b-3p was cloned into the AAV vector pAAV-CMV-ires-hrEGFP with $\mathrm{BamHI}$ and EcoRI restriction sites to construct the AAV transgene plasmid pAAV-CMV-miR-29b-3p-ires-hrEGFP. pAAV-RC9, pHelper, and the AAV transgene plasmid were co-transfected into HEK293 cells. At $72 \mathrm{~h}$ after transfection, the cells were incubated with Benzonase endonuclease (Sigma, USA) at $37^{\circ} \mathrm{C}$ for $1 \mathrm{~h}$, and then the AAV particles were purified by a heparin column (Sigma, USA). After the purification, the AAV particles were concentrated using Amicon Ultra-4 (Millipore, USA). AAV was aliquoted and stored at $-80^{\circ} \mathrm{C}$. The rats were anesthetized with sodium pentobarbital $(60 \mathrm{mg} / \mathrm{kg}$, intraperitoneally (i.p.)) and received injections into the PFC ( $+4.7 \mathrm{~mm} \mathrm{AP}, \pm 2 \mathrm{~mm} \mathrm{ML}$, and $-1.2 \mathrm{~mm} \mathrm{DV})$ using a stereotaxic frame (Kopf Instruments, CA, USA) linked to a digital rat brain atlas. Two microliters of AAV-miR29b-3p or AAV-GFP mixed with $1 \mu \mathrm{l}$ of $20 \%$ mannitol were injected at a speed of $0.1 \mu \mathrm{l} / \mathrm{min}$. The behavioral experiments were performed after 4 weeks to allow the rats injected with the AAVs to recover and the miRNA to be adequately expressed. The AAV infection effects were detected by immunofluorescence microscopy. The expression of miR-29b-3p and its target gene GRM4 in the rat PFC tissue was analyzed by qRT-PCR and Western blotting 28 days after the AAV infection.

\section{Cell viability assay}

The dissociated neuronal cells were seeded in poly-Dlysine-coated 96-well plates. The cells were maintained in a humidified atmosphere of $5 \% \mathrm{CO}_{2}$ at $37^{\circ} \mathrm{C}$ for 5 days and then infected with lenti-over/miR-29b-3p, lentiinhib/miR-29b-3p, or lenti-NC particles. After $72 \mathrm{~h}$, MTT (3-(4,5-dimethylthiazol-2-yl)-2,5-diphenyl tetrazolium bromide) reagent (Sigma, USA) was added to the medium, and the cells were incubated for $4 \mathrm{~h}$ before measuring the absorbance at $490 \mathrm{~nm}$.

\section{Extracellular and intracellular glutamate assay}

The extracellular and intracellular glutamate levels were measured using the BioVision's Glutamate Assay Kit. The dissociated neuronal cells were seeded in poly-Dlysine-coated 6-well plates and cultured for 3 days. Subsequently, the cells were infected with the lenti-over/ miR-29b-3p, lenti-inhib/miR-29b-3p, or lenti-NC 
particles. After $72 \mathrm{~h}$, the cell culture medium was directly diluted in the assay buffer for the extracellular glutamate determination. The cell numbers were normalized by the MTT assay. The cells $\left(1 \times 10^{6}\right)$ were homogenized in $100 \mu \mathrm{l}$ assay buffer for the intracellular glutamate assay. The glutamate levels were quantified by measuring the optical density at $\lambda=450 \mathrm{~nm}$ using a microplate reader. The final glutamate concentration was calculated according to standard curves constructed using known concentrations of exogenous glutamate.

\section{Calcium-sensitive fluorometric measurements}

The calcium imaging experiments were conducted using primary neuronal cells infected with the lenti-over/ miR-29b-3p, lenti-inhib/miR-29b-3p, and lenti-NC particles. After three washes with Hanks' balanced salt solution (HEPES buffer), the cells were treated with 5.0 $\mu \mathrm{M}$ Fura-2 AM (Netherlands) for $60 \mathrm{~min}$ at $37^{\circ} \mathrm{C}$ in a $\mathrm{CO}_{2}$ incubator. The cells were sequentially illuminated $(6 \mathrm{~s}$ each time for a total of $1 \mathrm{~min}$ ) at 340 and $380 \mathrm{~nm}$ (excitation wavelengths) under a laser scanning confocal microscope (Olympus, Japan). The calcium-dependent fluorescence signals were measured at $510 \mathrm{~nm}$ (emission wavelength). The relative intracellular $\mathrm{Ca}^{2+}$ level was determined using the formula $\triangle F=\left(F-F_{\min }\right) /\left(F_{\max }-F\right)$, where $F$ is the fluorescence intensity, $F_{\min }$ was determined by imaging the infected cells in a calcium-free saline solution, and $F_{\max }$ was determined by imaging the infected cells in normal saline solution with $5 \mathrm{M}$ ionomycin (Sigma-Aldrich, USA).

\section{Electrophysiological recordings}

The electrophysiological recordings were performed as previously described ${ }^{18}$. Briefly, the external solution used for $I_{\mathrm{Ca}}$ consisted of (in $\mathrm{mM}, \mathrm{pH}$ 7.4) choline-Cl 110, TEA$\mathrm{Cl}$ 20, 4-AP 10, $\mathrm{MgCl}_{2} 1, \mathrm{BaCl}_{2}$ 10, HEPES 10, glucose 10, and TTX $10^{-3}$. The internal solution used for $I_{\mathrm{Ca}}$ consisted of (in mM, pH 7.2) CsCl 110, TEA-Cl 30, EGTA 10, HEPES 10, and ATP-Mg3. The primary neuron cells were washed with bathing solution. The suspension of the neuronal cells was plated on the recording chamber of an inverted microscope (Olympus, Japan). A single neuron cell infected with a lentiviral particle was chosen. The whole-cell patch-clamp recording was performed with a patch-clamp amplifier. The acquisition, storage, and analysis of the current signals were performed by a MultiClamp 700B Patch-Clamp Amplifier (Axon Instruments), DigiData 1440A digital analog converter, and pClampex 10. 0 (Axon Instruments). The $I_{\mathrm{Ca}}(\mathrm{HVA})$ current (inward electric current) was recorded using stepwise $10-\mathrm{mV}$ polarizing pulses (200-ms duration) from a constant holding potential of $-50 \mathrm{mV}$ in the cells. The wholecell patch-clamp recordings of the lentivirus-infected PFC neuron cells were also performed after $30 \mathrm{~min}$ of incubation with drugs, including a GRM4 antagonist (MSOP,
$150 \mu \mathrm{M}), \mathrm{P} / \mathrm{Q}$-type $\mathrm{Ca}^{2+}$ channel blocker ( $\omega$-agatoxin TK, $250 \mathrm{nM}$ ), N-type $\mathrm{Ca}^{2+}$ channel blocker ( $\omega$-conotoxin GVIA, $250 \mathrm{nM}$ ), R-type $\mathrm{Ca}^{2+}$ channel blocker (SNX-482, $100 \mathrm{nM}$ ), phospholipase C (PLC) inhibitor (U73122, $1 \mu \mathrm{M}$ ), and protein kinase C inhibitor (GF109203X, $20 \mathrm{nM})$.

\section{Statistical analysis}

The Statistical Package for the Social Sciences (SPSS) version 16.0(SPSS Inc., Chicago, IL, USA) and GraphPad Prism 5.0 (San Diego, CA, USA) were used for the statistical analyses. All data are presented as the mean \pm SEM. Differences between two groups were determined by Student's $t$ test, and multigroup differences were determined by one-way analysis of variance, followed by Tukey's post hoc test. $P$ values $<0.05$ were considered statistically significant differences.

\section{Results}

Ketamine increased miR-29b-3p expression in the rat PFC

Recent studies suggest that miRNAs are associated with MDD pathogenesis ${ }^{28}$, and our previous reports show that miR-29a/b/c-3p was probably involved in MDD ${ }^{29}$. Ketamine administration is accepted as a rapid and persistent antidepressant treatment for $\mathrm{MDD}^{22,30,31}$. MDD is associated with altered neuronal and structural plasticity and neurogenesis in brain regions, including the hypothalamus, hippocampus, and PFC ${ }^{32-34}$. However, whether $\mathrm{miR}-29 \mathrm{a} / \mathrm{b} / \mathrm{c}-3 \mathrm{p}$ responds to ketamine treatment in the hypothalamus, hippocampus, and PFC is still unknown.

To determine the alteration in $\mathrm{miR}-29 \mathrm{a} / \mathrm{b} / \mathrm{c}-3 \mathrm{p}$ in the rat brain after ketamine $(10 \mathrm{mg} / \mathrm{kg})$ treatment, qRT-PCR was performed. The results showed that miR-29b-3p was increased in the PFC of the normal rats (Fig. 1b), but did not significantly change in the hypothalamus (Fig. 1a) or hippocampus (Fig. 1c). However, there was no significant change in miR-29a/c-3p in the PFC, hypothalamus, or hippocampus in the normal rats (Fig. 1b). These results indicate that miR-29b-3p is probably a ketamine-sensitive miRNA, especially in the PFC of normal rats.

\section{miR-29b-3p level in the PFC was decreased in the CUMS rats and restored by the ketamine treatment}

To further explore the alteration in miR-29b-3p in the hypothalamus, hippocampus, and PFC of depressed rats, CUMS was utilized to establish a rat depression model. In the behavioral tests, the rats exhibited depressive-like behaviors on the sucrose preference test $\left(F_{(3,18)}=27.710\right.$, $p<0.001)$ (Fig. 1d) and FST $\left(F_{(3,18)}=30.741, p<0.001\right)$ (Fig. 1e). Subsequently, the hypothalamus, hippocampus, and PFC of the depressed rats were isolated, and miR$29 \mathrm{~b}-3 \mathrm{p}$ expression was analyzed by qRT-PCR. The data indicated that miR-29b-3p was decreased by $21.6 \%$ $(t=4.463, p=0.004)$ in the PFC of the CUMS rat, but no 


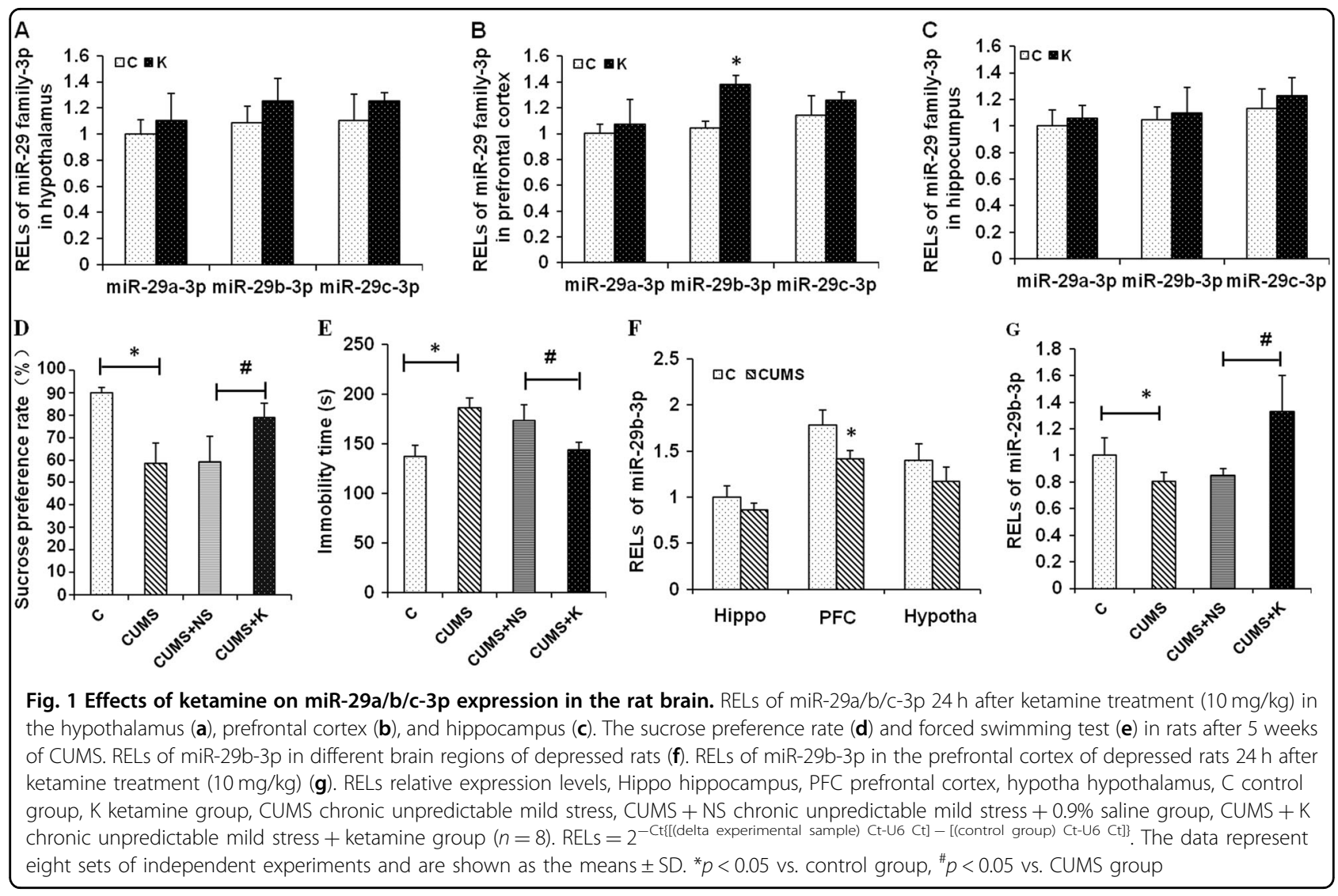

significant changes were observed in the hypothalamus $(t=2.143, \quad p=0.065)$ or hippocampus $(t=2.149$, $p=0.064$ ) (Fig. 1f). Compared with the saline-treated rats, after the ketamine administration, miR-29b-3p expression increased by $56.7 \%(t=-4.541, p=0.002)$ in the PFC of the CUMS rats (Fig. 1g). These results revealed that ketamine is able to promote miR-29b-3p expression in the PFC of CUMS rats, suggesting that miR-29b-3p is a possible key factor involved in ketamine's antidepressant effects.

\section{miR-29b-3p directly targeted GRM4}

miRNAs have been previously found to regulate distinct genes widely involved in neurodevelopmental and brain disorders $^{35}$. Therefore, we searched for the target genes of miR-29b-3p. The gene targets of miR-29b-3p were predicted using the following four miRNA target prediction databases: MicroCosm, miRanda, TargetScan, and miRDB. The results showed that GRM4 had a putative miR-29b-3p-binding site mapped to the $3^{\prime}$-UTR and was one of the high-scoring candidate genes for miR-29b-3p targeting (Fig. 2a). To test the effect of miR-29b-3p on GRM4 expression, lentiviruses overexpressing and silencing miR-29b-3p (named lenti-over/miR-29b-3p and lenti-inhib/miR-29b-3p) were used to infect primary neuron cells. miR-29b-3p was highly expressed after the
lenti-over/miR-29b-3p infection and downregulated after the lenti-inhib/miR-29b-3p infection (Fig. 2b). The expression of GRM4 in the infected cells was analyzed by Western blotting, and the results showed that GRM4 was negatively regulated by miR-29b-3p (Fig. 2c). These findings indicate that miR-29b-3p directly targets GRM4.

To further investigate whether miR-29b-3p directly targets GRM4, luciferase reporters containing either a wild-type GRM4 3'-UTR or a mutant GRM4 3'-UTR and mutant sequences of the miR-29b-3p-binding site were used (Fig. 2a). The results showed that compared with the control group, miR-29b-3p inhibited luciferase activity through the 3'-UTR of miR-29b-3p (Fig. 2d). The reporter activity of the GRM4 $3^{\prime}$-UTR containing a mutation in the miR-29b-3p-binding site did not significantly differ from that in the control transfected cells (Fig. 2d).

\section{Ketamine decreased GRM4 expression in primary neuron cells}

GRM4 has been implicated in the regulation of anxietyrelated behaviors ${ }^{19}$ and $\mathrm{MDD}^{23}$. However, whether ketamine influences the expression of GRM4 remains unknown. To validate the effect of ketamine-regulating GRM4 expression, rat PFC primary neuron cells were cultured. After the ketamine treatment, the qRT-PCR analysis showed that the GRM4 mRNA levels gradually 
declined at 1, 3, 6, and $12 \mathrm{~h}$ (Fig. 3b), while the miR-29b$3 p$ levels simultaneously increased (Fig. 3a), indicating that the miR-29b-3p levels were negatively correlated with GRM4 expression. The GRM4 protein levels were also detected by Western blotting and showed a trend similar to that of the mRNA levels (Fig. 3c). These results indicate that GRM4 expression in primary neuron cells is influenced by ketamine in a time-dependent manner.

\section{Ketamine mitigated the upregulation of GRM4 in the PFC of CUMS rats}

GRM4 has been reported to be upregulated in the PFC of MDD subjects ${ }^{36}$. Therefore, we examined the function of ketamine in the regulation of GRM4 expression in the PFC of depressed rats. GRM4 expression in the PFC of depressed rats was tested by qRT-PCR and Western blotting after a sub-anesthetic dosage of ketamine. Both
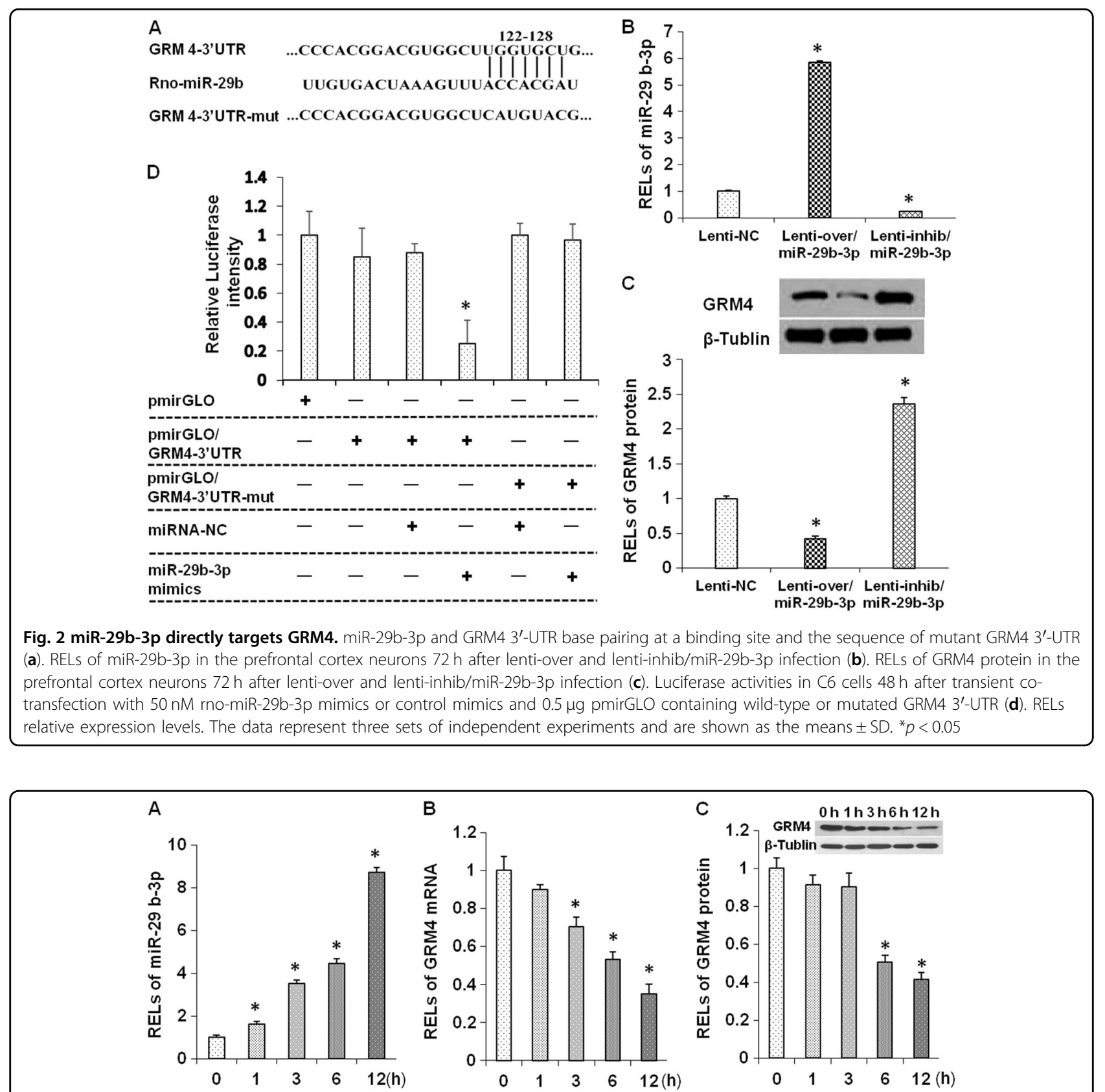

Fig. 3 Change in miR-29b-3p and GRM4 levels at different times after ketamine treatment in cultured prefrontal cortex neurons. RELs of miR-29b-3p (a) and GRM4 mRNA (b) in the prefrontal cortex neurons 1, 3, 6, and $12 \mathrm{~h}$ after a $50 \mu \mathrm{M}$ ketamine treatment. RELs of GRM4 protein in the prefrontal cortex neurons $1,3,6$, and $12 \mathrm{~h}$ after a $50 \mu \mathrm{M}$ ketamine treatment (c). RELs relative expression levels. The data represent three sets of independent experiments and are shown as the means \pm SD. ${ }^{*} p<0.05$ 
the mRNA and protein levels of GRM4 in the PFC were significantly increased in the CUMS rats (vs. the normal rats) and significantly decreased after the ketamine treatment (vs. the saline group) (Fig. 4), suggesting that ketamine could alleviate the upregulation of GRM4 in the PFC induced by CUMS.

Effects of miR-29b-3p on $\mathrm{Ca}^{2+}$ influx and glutamate release

Glutamate is a major excitatory neurotransmitter in the mammalian brain. Group III metabotropic glutamate receptors (mGluRs), including GRM4, negatively regulate $\mathrm{Ca}^{2+}$ influx into presynaptic terminals ${ }^{37}$ and thus decrease glutamatergic transmission ${ }^{38,39}$. To elucidate the effect of miR-29b-3p on $\mathrm{Ca}^{2+}$ influx, $\mathrm{Ca}^{2+}$ influx was examined by fluorometric calcium imaging and whole-cell patch-clamp recordings. As shown in Fig. 5a, the downregulation of miR-29b-3p by lenti-inhib/miR-29b-3p decreased the intracellular $\mathrm{Ca}^{2+}$ content, and the upregulation of miR-29b-3p by lenti-over/miR-29b-3p increased the intracellular $\mathrm{Ca}^{2+}$ content. As shown by the electrophysiology analysis, the overexpression of miR29b-3p significantly enforced the $I_{\mathrm{Ca}}$ (HVA) currents (Fig. 5b); the pharmacological agonist of mGluR4 had been proved to acutely inhibit the presynaptic calcium $\left(\mathrm{Ca}^{2+}\right)$ influx that ultimately controls glutamate release ${ }^{40}$. Therefore, we used the antagonist MSOP in this experiment. However, unexpectedly, the group III mGluR antagonist (MSOP, $150 \mu \mathrm{M}$ ) treatment in the cells overexpressing miR-29b-3p also inhibited the presynaptic calcium $\left(\mathrm{Ca}^{2+}\right)$ influx (Fig. 5b).

Presynaptic terminal N-type, P/Q-type, and R-type voltage-dependent calcium channels (VDCCs) play an important role in $\mathrm{Ca}^{2+}$ influx ${ }^{37}$. The modulating effect of miR-29b-3p on VDCCs was analyzed, and the results showed that P/Q-type, N-type, and R-type VDCC blocker incubation decreased the $I_{\mathrm{Ca}}$ (HVA) current promoted by the lenti-over/miR-29b-3p infection (Fig. 5b). Meanwhile, PLC-PKC (protein kinase C) ${ }^{38,39}$ is regarded as an important signaling cascade promoting $\mathrm{Ca}^{2+}$ influx. To determine the involvement of miR-29b-3p in PLC-PKC signaling, the inhibitors shown in Fig. 5b were used. The results showed that both the PLC and PKC inhibitors decreased the $I_{\mathrm{Ca}}(\mathrm{HVA})$ current promoted by the lentiover/miR-29b-3p infection.

Subsequently, the influence of miR-29b-3p on glutamate release was examined. The cytoplasm and extracellular glutamate levels in the lenti-over/miR-29b-3pinfected and lenti-inhib/miR-29b-3p-infected primary neuron cells was analyzed by a Glutamate Assay Kit. Our data showed that the high miR-29b-3p expression decreased the cytoplasm glutamate concentration, while the decreased miR-29b-3p expression showed the opposite effect (Fig. 5c). As shown in Fig. 5d, the extracellular glutamate levels were increased by the miR-29b-3p overexpression and decreased by the silencing of miR29b-3p. These findings suggest that miR-29b-3p likely promotes $\mathrm{Ca}^{2+}$ influx and then increases glutamatergic transmission by targeting GRM4.

\section{miR-29b-3p promoted cell survival and cytodendrite growth}

Brain imaging and postmortem studies suggest that reductions in dendrite arborization and complexity and the number of neurons and glia in brain regions, including the hippocampus, PFC, cingulate cortex, nucleus accumbens, and amygdala, could contribute to depressive symptoms $^{32-34}$. Therefore, we investigated the function of miR-29b-3p in neuron cell survival and cytodendrite
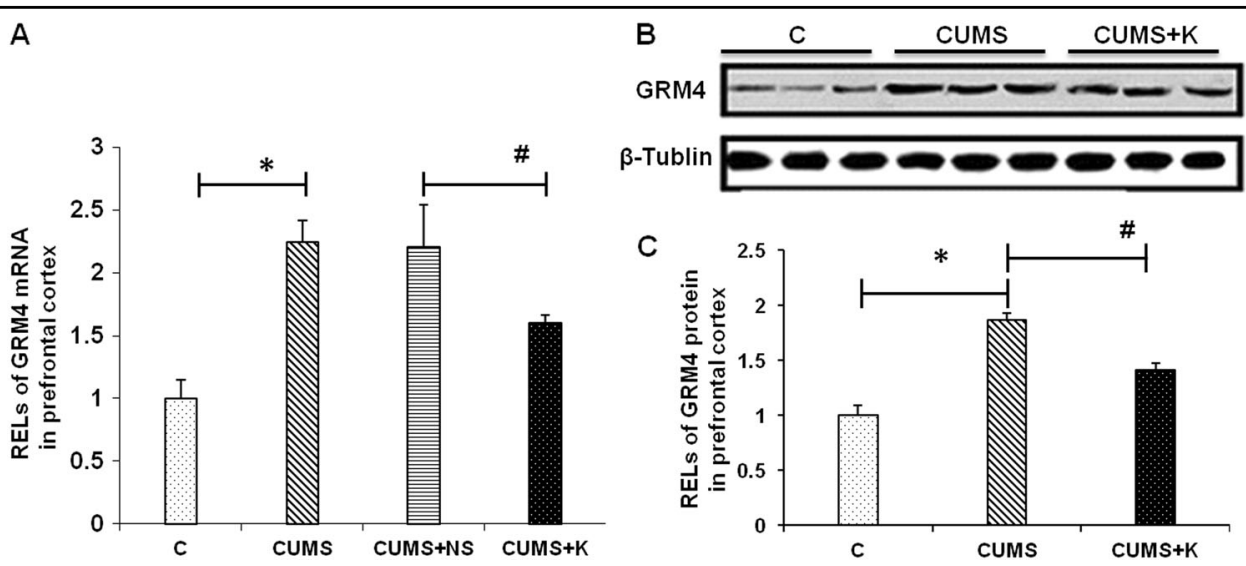

Fig. 4 Change in GRM4 expression levels in the prefrontal cortex of depressed rats following ketamine treatment. RELs of GRM4 mRNA in the prefrontal cortex of depressed rats $24 \mathrm{~h}$ after a $10 \mathrm{mg} / \mathrm{kg}$ ketamine treatment (a). RELs of GRM4 protein in the prefrontal cortex of depressed rats $24 \mathrm{~h}$ after a $10 \mathrm{mg} / \mathrm{kg}$ ketamine treatment $(\mathbf{b}, \mathbf{c})$. C control group, CUMS chronic unpredictable mild stress, CUMS + NS CUMS + 0.9\% saline group, CUMS + K CUMS + ketamine group, RELs relative expression levels. The data represent eight sets of independent experiments and are shown as the means \pm SD. ${ }^{*} p<0.05$ vs. control group, ${ }^{*} p<0.05$ vs. CUMS group 


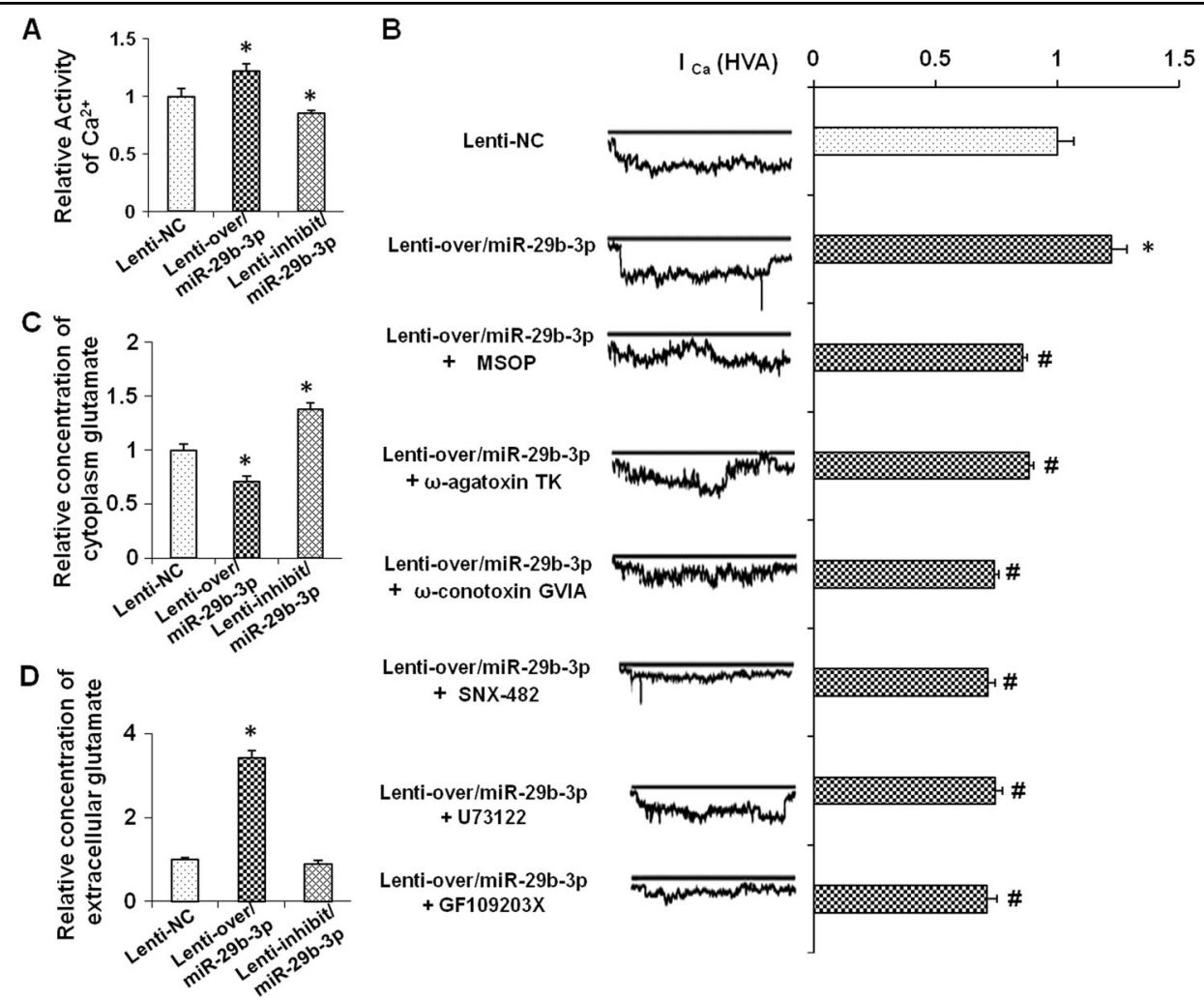

Fig. 5 Effects of miR-29b-3p on $\mathrm{Ca}^{2+}$ influx and glutamate release. The relative $\mathrm{Ca}^{2+}$ activity in the prefrontal cortex neurons $72 \mathrm{~h}$ after lenti-over and lenti-inhib/miR-29b-3p infection (a). I Ca (HVA) current (inward electric current) in cells infected by lenti-over/miR-29b-3p or treated with a GRM4 antagonist (MSOP, $150 \mu \mathrm{M}$ ), a P/Q-type $\mathrm{Ca}^{2+}$ channel blocker ( $\omega$-agatoxin TK, $250 \mathrm{nM}$ ), N-type $\mathrm{Ca}^{2+}$ channel blocker ( $\omega$-conotoxin GVIA, 250 nM), R-

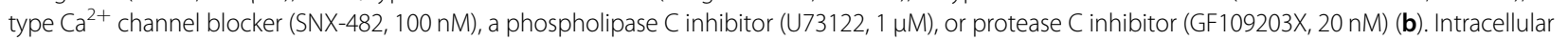
(c) and extracellular (d) glutamate concentrations $72 \mathrm{~h}$ after lenti-over/miR-29b-3p and lenti-inhib/miR-29b-3p infection. The data represent three sets of independent experiments and are shown as the means \pm SD. ${ }^{*} p<0.05$ vs. lenti-NC; ${ }^{*} p<0.05$ vs. lenti-over/miR-29b-3p

growth. The cell viability as determined by an MTT assay was increased by $39 \%$ after the lenti-over/miR-29b-3p infection and decreased by $41 \%$ after the lenti-inhib/miR29b-3p infection (Fig. 6a). Cell apoptosis, which was tested by TUNEL staining, was elevated by approximately 3-fold after the miR-29b-3p silencing, but no significant changes were found in the miR-29b-3p-overexpressing cell group (Fig. 6b). Cytodendrite growth was observed with immunofluorescence microscopy and found to be promoted in the lenti-over/miR-29b-3p-infected neuron cells, while no significant changes were found in the lentiinhib/miR-29b-3p-infected neuron cells (Fig. 6c). These results indicate that miR-29b-3p benefits cell growth, cell survival, and cytodendrite growth.

\section{miR-29b-3p overexpression improved depressive behaviors in CUMS rats}

To further determine whether miR-2b-3p could target GRM4 in rat PFC tissue and mitigate the depressive behaviors of depressed rats, AAV-miR-29b-3p was injected into the PFC of CUMS rats using a stereotaxic frame. After 28 days, the AAV effectively infected the brain tissue as indicated by immunofluorescence microscopy (Fig. 7a). Saline and AAV-GFP served as controls.

To examine the effects of miR-29b-3p on major depression disorder, AAV-miR-29b-3p-infected depressive-like rats were subjected to behavioral tests, including a sucrose preference test, open-field test, and FST. The AAV-miR$29 \mathrm{~b}-3 \mathrm{p}$ infection led to increased sucrose preference $\left(F_{(2,15)}\right.$ $=32.466, p<0.001$ ) (Fig. 7b) and shortened immobility time in the FST $\left(F_{(2,15)}=58.113, p<0.001\right)$ (Fig. 7c), indicating that miR-29b-3p has an antidepressant effect.

The RT-qPCR analysis showed that the level of miR29b-3p dramatically increased after the AAV-miR-29b-3p infection to 4.42 times that in the AAV-GFP group (Fig. 7d), while GRM4 expression decreased by $56.1 \%$ (Fig. 7e). The GRM4 protein levels in the rat PFC were also detected by Western blotting and showed a significant decrease after the AAV-miR-29b-3p infection (Fig. 7f).

\section{Discussion}

Our study uncovers a novel signaling pathway involved in the ketamine-induced alterations in MDD, implicating 


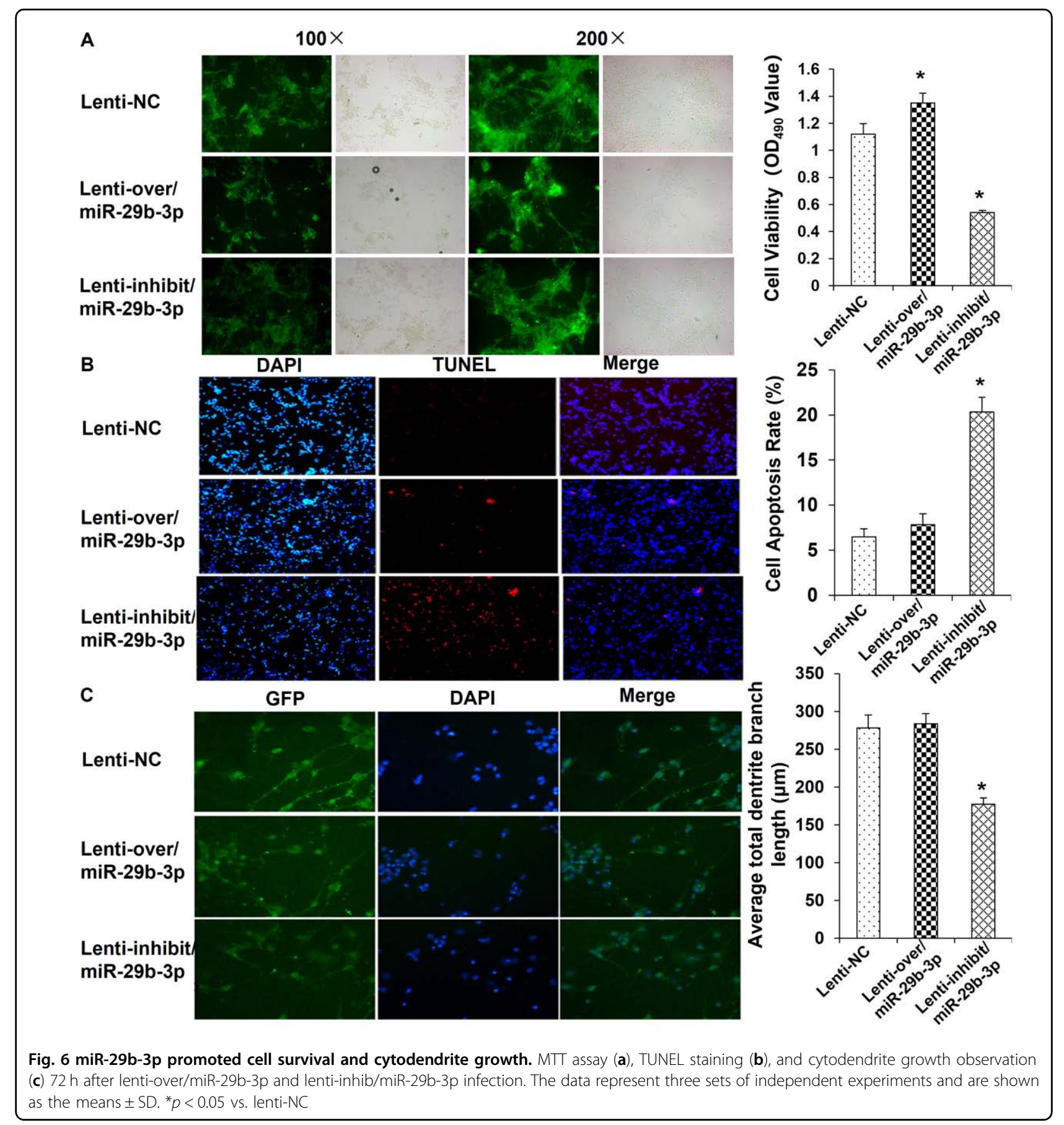

both upstream and downstream effectors of miR-29b-3p. The miR-29b-3p/GRM4 pathway is a critical mediator of ketamine's antidepressant function in depressive-like rats, and the miR-29b-3p/GRM4 pathway could be considered a potential therapeutic target, opening up novel avenues for drug design for MDD treatment.

MDD is a prevalent central nervous system disorder associated with differential brain regions, including the hypothalamus, hippocampus, and $\mathrm{PFC}^{32-34}$. According to recent research, ketamine has a rapid and persistent antidepressant effect $^{3-5}$ and influences the hypothalamus $^{41}$, hippocampus ${ }^{18}$, and $\mathrm{PFC}^{42}$. O'Connor et al. ${ }^{11}$ found that miR-29b could be upregulated by ketamine treatment in the hippocampus in an early depression rat model. However, in this study, we found a slight, nonsignificant increase in miR-29b-3p in the hippocampus of normal rats after acute ketamine administration and that miR-29b-3p may be more sensitive to ketamine treatment 


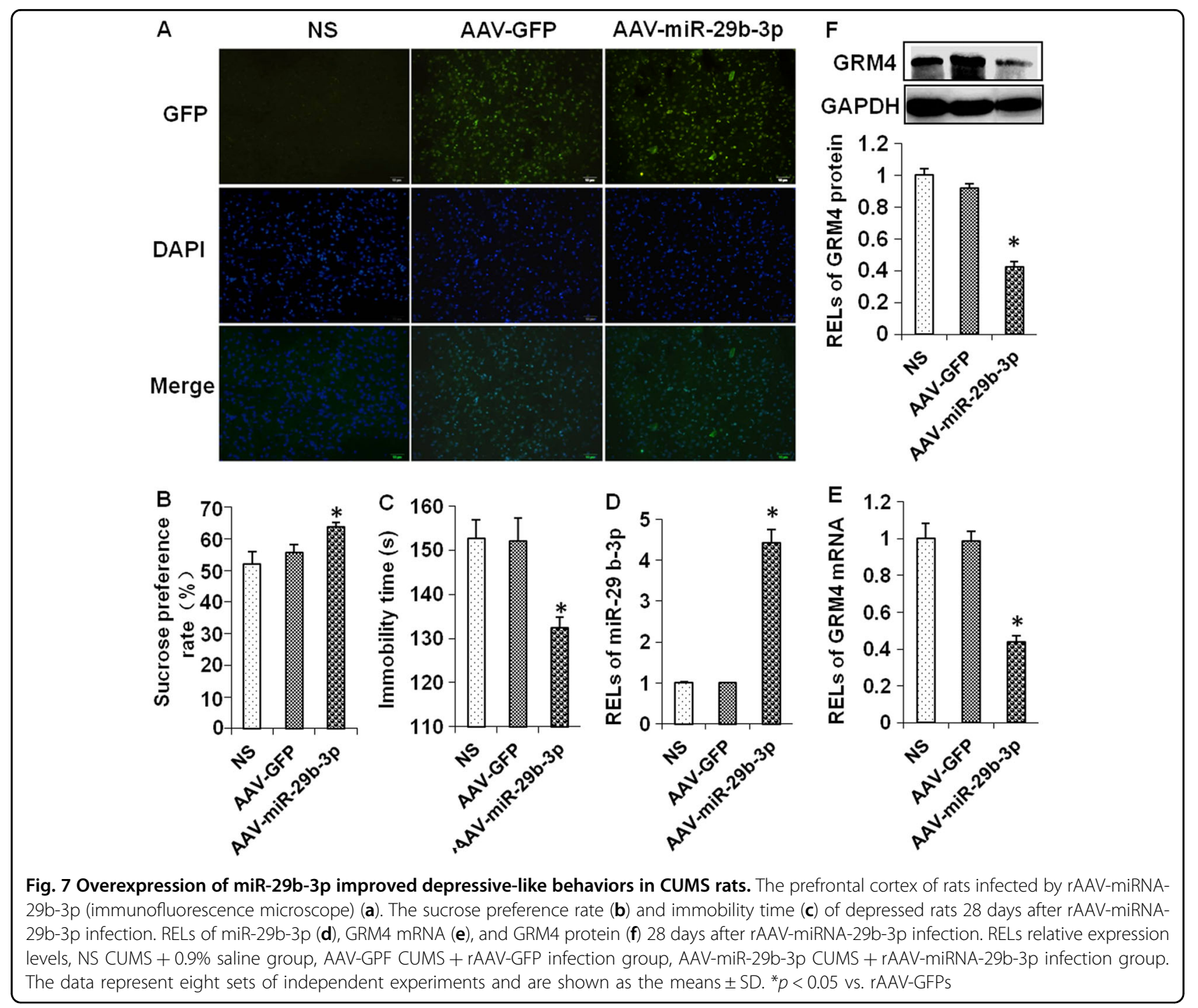

in depressed animals than normal animal. Interestingly, our results demonstrated that miR-29b-3p expression could be upregulated by ketamine in the rat PFC. The miR-29b-3p levels gradually increased $1,3,6$, and $12 \mathrm{~h}$ post ketamine incubation, which is consistent with the rapid and persistent antidepressant effects of ketamine. Hence, the corresponding action of ketamine is achieved through miR-29b-3p, which may be a key factor in ketamine's antidepressant effects. However, the mechanism by which miR-29b-3p is upregulated by ketamine is still unclear. miR-29 expression has been previously reported to be repressed by nuclear factor $-\mathrm{K} B(\mathrm{NF}-\mathrm{k} B)$ signaling, and NF- $k B$ downregulation resulted in a depression ${ }^{43}$, while ketamine could suppress NF- $\kappa B$ activity ${ }^{44}$; therefore, ketamine possibly upregulates miR-29b-3p by inhibiting NF- $\mathrm{BB}$ activity. Then, CUMS was used to establish a depression model, and miR-29b-3p expression was analyzed by qRT-PCR. Interestingly, the results showed that miR-29b-3p was decreased in the PFC of the depressive rats, whereas after the ketamine administration, miR-29b-3p was increased in the PFC of the depressive rats. Therefore, miR-29b-3p is involved in depression and ketamine's antidepressant effects.

However, the downstream mechanism of miR-29b-3p in the progression of depression and ketamine's antidepressant effects remains unclear. Using the target prediction databases MicroCosm, miRanda, TargetScan, and miRDB, we found that GRM4 may be the downstream target gene of miR-29b-3p. The luciferase reporters also confirmed that miR-29b-3p directly targets GRM4 and that GRM4 was negatively regulated by miR-29b-3p. In addition, Lopez et al. ${ }^{36}$ and $\mathrm{Li}$ et al. ${ }^{20}$ found that GRM4 was increased in PFC samples and blood samples from depressed patients, and GRM4 has also been implicated in the regulation of anxiety-related behaviors ${ }^{19}$. Therefore, GRM4 was regarded as a potential therapeutic target 
gene. Although there is no direct evidence that GRM4 modulates depression-like behaviors based on studies investigating GRM4-overexpressed or GRM4-knockdown animal models, GRM4 has been implicated in the regulation of MDD and is considered an attractive target for drug discovery. In our future research, we aim to further study the function of GRM4 in modulating depressionlike behaviors and mediating the antidepressant effects of ketamine in GRM4-overexpressed or GRM4-knockdown animal models. However, whether GRM4 participates in the antidepressant activity of ketamine is unclear. In our study, GRM4 expression was increased in the PFC of depressed rats established by CUMS, and the GRM4 levels were decreased in both the PFC and primary neuron cells after the ketamine treatment, supporting the assumption that GRM4 is a ketamine-sensitive gene. Concomitantly, miR-29b-3p was upregulated by ketamine, negatively correlated with the GRM4 levels, and predicted to be a direct regulator of GRM4, which was further supported by the luciferase reporter assay. miR- $1202^{36}$ and miR- $355^{20}$ have also been found to regulate GRM4 expression, but whether ketamine treatment alters the expression levels of these miRNAs requires further investigation. Based on the miRNA array analysis in our previous study ${ }^{18}$, multiple miRNAs are aberrantly expressed in the rat hippocampus after ketamine administration, and these miRNAs did not include miR-1202 or miR-355. Whether miR-1202 and miR-355 in the PFC and other brain regions respond to ketamine treatment has not been previously studied. In this study, we overexpressed miR-29b-3p in the PFC of depressed rats using the AAV infection technique and determined that miR-29b-3p downregulated the expression of GRM4, suggesting that miR-29b-3p regulates GRM4 expression both in vitro and in vivo. Therefore, GRM4 plays an important role in the downstream mechanism of miR-29b-3p in the progression of depression and ketamine's antidepressant effects.

miR-29b-3p is involved in the antidepressive-like actions of ketamine, but to strengthen this main conclusion, additional data should be obtained by examining whether the antidepressive-like effects of ketamine are affected in animal models in which miR-29b-3p is overexpressed or knocked down in vivo. Therefore, miR-29b3p-overexpressing and miR-29b-3p-knockdown animal testing will be performed in the future.

\section{miR-29b-3p contributes to $\mathrm{Ca}^{2+}$ influx in a GRM4- dependent manner}

L-Glutamate is a major excitatory neurotransmitter in the central nervous system and activates both ionotropic and metabotropic glutamate receptors. Glutamatergic neurotransmission is involved in most aspects of normal brain functioning and can be perturbed in many neuropathological conditions. GRM4 belongs to metabotropic glutamate receptor group III and is mainly located on the presynaptic membrane, inhibiting $\mathrm{Ca}^{2+}$ influx into presynaptic terminals and, thus, decreasing glutamatergic transmission ${ }^{40}$. We examined the effect of miR-29b-3p on $\mathrm{Ca}^{2+}$ influx in this study. The calcium imaging showed that miR-29b-3p overexpression increased the intracellular $\mathrm{Ca}^{2+}$ concentration, and the whole-cell patch-clamp recording showed that miR-29b-3p overexpression promoted $I_{\mathrm{Ca}}$ currents. These results indicate that the overexpression of miR-29b-3p targeting GRM4 contributes to $\mathrm{Ca}^{2+}$ influx. However, unexpectedly, the MOSP treatment of cells overexpressing miR-29b-3p also inhibited the influx of presynaptic calcium $\left(\mathrm{Ca}^{2+}\right)$. One reason may be that MOSP is a selective antagonist of group III mGluRs, which include the following four subtypes: mGlu4, mGlu6, mGlu7, and mGlu8; compared to mGlu4, the other subtypes probably show different effects on calcium $\left(\mathrm{Ca}^{2+}\right)$ influx. For example, mGlu7 and mGlu8 mediated opposite effects in several studies ${ }^{45,46}$.

The types of presynaptic voltage-gated calcium channels (VGCCs) modulated by miR-29b-3p require further exploration. Our data showed that the overexpression of miR-29-3p increased the $\mathrm{Ca}^{2+}$ influx, but was totally inhibited by any type of VGCC, indicating that miR-29-3p is probably involved in VDCC-mediated $\mathrm{Ca}^{2+}$ influx, which is consistent with the modulating effect of GRM4 on multiple types of voltage-gated $\mathrm{Ca}^{2+}$ channels ${ }^{47}$. The PLC or PKC inhibitor could reverse the increased $\mathrm{Ca}^{2+}$ influx induced by miR-29-3p, suggesting that miR-29-3p could influence $\mathrm{Ca}^{2}$ ${ }^{+}$influx through a PLC-PKC signaling cascade, which is consistent with the role of GRM4 in depressing the presynaptic calcium influx via a PLC-PKC signaling pathway ${ }^{48}$.

Our study also found that miR-29b-3p promotes glutamate release. miR-29b-3p's promotion of $\mathrm{Ca}^{2+}$ influx and glutamate release may explain why the miR-29-3p infection led to improvement in the depressive behaviors of the depressed rats. Additionally, miR-29b-3p was found to benefit neuron cell survival and cytodendrite growth in the in vitro cultured neuron model, which could be another reason explaining miR-29b-3p's beneficial effects on depression. The pharmacological effects of ketamine have been proved to increase mammalian target of rapamycin complex 1 signaling by activating threonine kinase and increasing synaptic number and function in the PFC in vivo ${ }^{49,50}$. In this study, the effects of miR-29b-3p in the in vitro cultured neuron model showed similar results. However, whether these effects are GRM4-dependent remains unclear, and further investigation of relevant novel target genes is essential.

The depressive-like behaviors of the CUMS rats were alleviated by the overexpression of miR-29b-3p in the PFC via AAV infection, but the CUMS rats overexpressing miR-29b-3p significantly differed from the normal rats in the behaviors tests $(p=0.002)$ and FST $(p=0.007)$. These 
results suggest that the overexpression of miR-29b-3p did not totally reverse the depressive-like behaviors of the CUMS rats. Therefore, further studies are required to uncover the complex mechanisms of MDD.

In conclusion, miR-29b-3p was downregulated in the PFC of the depressed rats and upregulated by the ketamine treatment. GRM4 expression was negatively regulated by ketamine and miR-29b-3p. The miR-29b-3p overexpression contributed to $\mathrm{Ca}^{2+}$ influx, glutamate release, neuron cell survival, and cytodendrite growth and subsequently improved the depressive behaviors of depressed rats, representing a potential novel molecular mechanism underlying ketamine's antidepressant effect.

\section{Acknowledgements}

This work was supported by the Projects of the National Natural Science Foundation of China (Grant No. 81271478), Department of Science and Technology of Sichuan Province (Grant No. 14JC0093), Department of Education of Sichuan Province (Grant No. 14ZA0142), and Department of Science and Technology of Luzhou City (Grant No. 2015-S-46) to X.B.W. We would like to thank LetPub (www.letpub.com) for providing linguistic assistance during the preparation of this manuscript.

\section{Author details}

'Department of Anesthesiology, The Affiliated Hospital of Southwest Medical University, Luzhou, Sichuan Province, People's Republic of China. ${ }^{2}$ Laboratory of Anesthesiology, The Affiliated Hospital of Southwest Medical University, Luzhou, Sichuan Province, People's Republic of China. ${ }^{3}$ Department of Intensive Care Unit, The Affiliated Chaoyang Hospital of Capital Medical University, Beijing, People's Republic of China. ${ }^{4}$ Department of Biomedical Engineering, School of Medicine, University of Alabama at Birmingham, Birmingham, AL, USA

\section{Conflict of interest}

The authors declare that they have no conflict of interest.

\section{Publisher's note}

Springer Nature remains neutral with regard to jurisdictional claims in published maps and institutional affiliations.

Supplementary information accompanies this paper at https://doi.org/ 10.1038/s12276-018-0164-4.

Received: 7 February 2018 Revised: 25 June 2018 Accepted: 28 June 2018. Published online: 29 October 2018

\section{References}

1. Ferrari, A. J. et al. Burden of depressive disorders by country, sex, age, and year: findings from the global burden of disease study. PLoS Med. 10, e10015472013 (2010).

2. Murray, C. J. \& Lopez, A. D. Alternative projections of mortality and disability by cause 1990-2020: Global Burden of Disease Study. Lancet 349, 1498-1504 (1997).

3. Yang, C., Zhou, Z. Q., Gao, Z. Q., Shi, J. Y. \& Yang, J. J. Acute increases in plasma mammalian target of rapamycin, glycogen synthase kinase-3beta, and eukaryotic elongation factor 2 phosphorylation after ketamine treatment in three depressed patients. Biol. Psychiaty 73, e35-e36 (2013).

4. Wang, X. et al. Effects of propofol and ketamine as combined anesthesia for electroconvulsive therapy in patients with depressive disorder. J. ECT $\mathbf{2 8}$, 128-132 (2012)

5. Sun, H. L. et al. Role of hippocampal p11 in the sustained antidepressant effect of ketamine in the chronic unpredictable mild stress model. Transl. Psychiatry 6, e741 (2016).
6. Zhang, J. C., Li, S. X. \& Hashimoto, K. R (-)-ketamine shows greater potency and longer lasting antidepressant effects than $S(+)$-ketamine. Pharmacol. Biochem. Behav. 116, 137-141 (2014).

7. Yang, J. J. et al. Serum interleukin-6 is a predictive biomarker for ketamine's antidepressant effect in treatment-resistant patients with major depression. Biol. Psychiatry 77, e19-e20 (2015).

8. Baudry, A., Mouillet-Richard, S., Schneider, B., Launay, J. M. \& Kellermann, O. miR-16 targets the serotonin transporter: a new facet for adaptive responses to antidepressants. Science 329, 1537-1541 (2010).

9. Issler, O. et al. MicroRNA 135 is essential for chronic stress resiliency, antidepressant efficacy, and intact serotonergic activity. Neuron 83, 344-360 (2014).

10. Lopez, J. P. et al. MicroRNAs $146 a / b-5$ and $425-3 p$ and $24-3 p$ are markers of antidepressant response and regulate MAPKWnt-system genes. Nat. Commun. 8, 15497 (2017).

11. O'Connor, R. M., Grenham, S., Dinan, T. G. \& Cryan, J. F. microRNAs as novel antidepressant targets: converging effects of ketamine and electroconvulsive shock therapy in the rat hippocampus. Int. J. Neuropsychopharmacol. 16, 1885-1892 (2013).

12. Babenko, O., Golubov, A., Inytskyy, Y., Kovalchuk, I. \& Metz, G. A. Genomic and epigenomic responses to chronic stress involve miRNA-mediated programming. PLoS ONE 7, e29441 (2012).

13. Jin, X. F., Wu, N., Wang, L. \& Li, J. Circulating microRNAs: a novel class of potential biomarkers for diagnosing and prognosing central nervous system diseases. Cell. Mol. Neurobiol. 33, 601-613 (2013).

14. Zong, Y. et al. miR-29c regulates NAV3 protein expression in a transgenic mouse model of Alzheimer's disease. Brain Res. 1624, 95-102 (2015).

15. Bai, X. et al. Downregulation of blood serum microRNA 29 family in patients with Parkinson's disease. Sci. Rep. 7, 5411 (2017).

16. Yang, G. et al. MicroRNA-29c targets beta-site amyloid precursor proteincleaving enzyme 1 and has a neuroprotective role in vitro and in vivo. Mol. Med. Rep. 12, 3081-2088 (2015).

17. Nolan, $\mathrm{K}$. et al. Increased expression of microRNA-29a in ALS mice: functional analysis of its inhibition. J. Mol. Neurosci. 53, 231-241 (2014).

18. Yang, X. et al. MicroRNA expression profile and functional analysis reveal that miR-206 is a critical novel gene for the expression of BDNF induced by ketamine. Neuromol. Med. 16, 594-605 (2014).

19. Davis, M. J. et al. Role of mGluR4 in acquisition of fear learning and memory. Neuropharmacology 66, 365-372 (2013).

20. Li, J., Meng, H., Cao, W. \& Qiu, T. MiR-335 is involved in major depression disorder and antidepressant treatment through targeting GRM4. Neurosci. Lett. 606, 167-172 (2015).

21. Reus, G. Z. et al. Ketamine plus imipramine treatment induces antidepressantlike behavior and increases CREB and BDNF protein levels and PKA and PKC phosphorylation in rat brain. Behav. Brain. Res. 221, 166-171 (2011).

22. Garcia, L. S. et al. Acute administration of ketamine induces antidepressant-like effects in the forced swimming test and increases BDNF levels in the rat hippocampus. Prog. Neuropsychopharmacol. Biol. Psychiatry 32, 140-144 (2008).

23. Surget, A. et al. Corticolimbic transcriptome changes are state-dependent and region-specific in a rodent model of depression and of antidepressant reversal. Neuropsychopharmacology 34, 1363-1380 (2009).

24. Ducottet, C., Griebel, G. \& Belzung, C. Effects of the selective nonpeptide corticotropin-releasing factor receptor 1 antagonist antalarmin in the chronic mild stress model of depression in mice. Prog. Neuropsychopharmacol. Biol. Psychiatry 27, 625-631 (2003).

25. Iniguez, S. D. et al. Social defeat stress induces a depression-like phenotype in adolescent male c57BL/6 mice. Stress 17, 247-255 (2014).

26. Fath, T., Ke, Y. D., Gunning, P., Gotz, J. \& Ittner, L. M. Primary support cultures of hippocampal and substantia nigra neurons. Nat. Protoc. 4, 78-85 (2009).

27. Wang, $X$. et al. Unexpected pro-injury effect of propofol on vascular smooth muscle cells with increased oxidative stress. Crit. Care Med. 39, 738-745 (2011).

28. Dwivedi, Y. Emerging role of microRNAs in major depressive disorder: diagnosis and therapeutic implications. Dialog. Clin. Neurosci. 16, 43-61 (2014).

29. Wan, Y. et al. Identification of differential microRNAs in cerebrospinal fluid and serum of patients with major depressive disorder. PLOS ONE 10, e0121975 (2015).

30. Aan Het Rot, M., Zarate, C. A., Charney, D. S. \& Mathew, S. J. Ketamine for depression: where do we go from here? Biol. Psychiatry 72, 537-547 (2012).

31. Aan het Rot, M. et al. Safety and efficacy of repeated-dose intravenous ketamine for treatment-resistant depression. Biol. Psychiatry 67, 139-145 (2010). 
32. Yang, R. et al. Hypothalamus-anchored resting brain network changes before and after sertraline treatment in major depression. Biomed. Res. Int. 2014 915026 (2014).

33. Mahajan, G. J. et al. Altered neuro-inflammatory gene expression in hippocampus in major depressive disorder. Prog. Neuropsychopharmacol. Biol. Psychiatry 82, 177-186 (2018).

34. Shan, Y. et al. Correlations between working memory impairment and neurometabolites of prefrontal cortex and lenticular nucleus in patients with major depressive disorder. J. Affect. Disord. 227, 236-242 (2017).

35. Dwivedi, Y. Evidence demonstrating role of microRNAs in the etiopathology of major depression. J. Chem. Neuroanat. 42, 142-156 (2011).

36. Lopez, J. P. et al. miR-1202 is a primate-specific and brain-enriched microRNA involved in major depression and antidepressant treatment. Nat. Med. 20 764-768 (2014).

37. Zhang, W. \& Linden, D. J. Neuromodulation at single presynaptic boutons of cerebellar parallel fibers is determined by bouton size and basal action potential-evoked Ca transient amplitude. J. Neurosci. 29, 15586-15594 (2009).

38. Lorez, M., Humbel, U., Pflimlin, M. C. \& Kew, J. N. Group III metabotropic glutamate receptors as autoreceptors in the cerebellar cortex. Br. J. Pharmacol. 138, 614-625 (2003)

39. Pekhletski, R. et al. Impaired cerebellar synaptic plasticity and motor performance in mice lacking the mGluR4 subtype of metabotropic glutamate receptor. J. Neurosci. 16, 6364-6373 (1996).

40. Abitbol, K., McLean, H., Bessiron, T. \& Daniel, H. A new signalling pathway for parallel fibre presynaptic type 4 metabotropic glutamate receptors (mGluR4) in the rat cerebellar cortex. J. Physiol. 590, 2977-2994 (2012).
41. Niesters, M., Martini, C. \& Dahan, A. Ketamine for chronic pain: risks and benefits. Br. J. Clin. Pharmacol. 277, 357-367 (2014).

42. Liu, R. J. et al. GSK-3 inhibition potentiates the synaptogenic and antidepressant-like effects of subthreshold doses of ketamine. Neuropsychopharmacology 38, 2268-2277 (2013).

43. Wang, $H$. et al. NF-kappaB-YY1-miR-29 regulatory circuitry in skeletal myogenesis and rhabdomyosarcoma. Cancer Cell 14, 369-381 (2008).

44. Yu, M., Shao, D., Yang, J., Feng, S. \& Xu, J. Ketamine suppresses intestinal TLR4 expression and NF-kappaB activity in lipopolysaccharide-treated rats. Croat. Med. J. 47, 825-831 (2006).

45. Sun, $\mathrm{N}$. et al. Ventrolateral periaqueductal gray metabotropic glutamate receptor subtypes 7 and 8 mediate opposite effects on cardiosomatic motor reflex in rats. Nanfang Yi Ke Da Xue Xue Bao 34, 8-13 (2014).

46. Liu, X. H. et al. Metabotropic glutamate subtype 7 and 8 receptors oppositely modulate cardiac nociception in the rat nucleus tractus solitarius. Neuroscience 220, 322-329 (2012).

47. Jin, W., Lo, T. M., Loh, H. H. \& Thayer, S. A. U73122 inhibits phospholipase Cdependent calcium mobilization in neuronal cells. Brain Res. 642, 237-243 (1994).

48. Levin, R., Braiman, A. \& Priel, Z. Protein kinase C induced calcium influx and sustained enhancement of ciliary beating by extracellular ATP. Cell Calcium 21, 103-113 (1997).

49. Gerhard, D. M., Wohleb, E. S. \& Duman, R. S. Emerging treatment mechanisms for depression: focus on glutamate and synaptic plasticity. Drug Discov. Today 21, 454-464 (2016).

50. Belujon, P. \& Grace, A. A. Restoring mood balance in depression: ketamine reverses deficit in dopamine-dependent synaptic plasticity. Biol. Psychiatry 76, 927-936 (2014) 